\title{
Lidil
}

Revue de linguistique et de didactique des langues

43 | 2011

Le rapport au savoir dans les discours professionnels

\section{Rapports aux textes virtuels en formation de lettres : vers une nouvelle écriture professionnelle}

The position of Literacy teachers with the Hypertexts: new gestures for the professional?

\section{Véronique Rey et Céline Beaudet}

\section{(2) OpenEdition}

\section{Journals}

Édition électronique

URL : http://journals.openedition.org/lidil/3114

DOI : 10.4000/lidil.3114

ISSN : $1960-6052$

Éditeur

UGA Éditions/Université Grenoble Alpes

Édition imprimée

Date de publication : 30 mai 2011

Pagination : 103-116

ISBN : 978-2-84310-201-1

ISSN : $1146-6480$

\section{Référence électronique}

Véronique Rey et Céline Beaudet, «Rapports aux textes virtuels en formation de lettres : vers une nouvelle écriture professionnelle », Lidil [En ligne], 43 | 2011, mis en ligne le 30 novembre 2012 consulté le 01 mai 2019. URL : http://journals.openedition.org/lidil/3114 ; DOI : 10.4000/lidil.3114 


\title{
Rapports aux textes virtuels en formation de lettres : vers une nouvelle écriture professionnelle
}

\author{
Véronique Rey* et Céline Beaudet**
}

\begin{abstract}
RÉSUMÉ
La littératie étendue correspond à des écritures générant la gestion de plusieurs contraintes. Les textes numérisés et les hypertextes exigent du scripteur des macrohabiletés textuelles et discursives qui nécessitent des formations. Un rapide historique rappelle les éléments principaux de cette révolution et leurs conséquences dans le domaine scolaire. La rupture est également abordée en interrogeant les enjeux pragmatiques et énonciatifs des hypertextes. Les comportements des enseignants de lettres face à cette nouvelle réalité professionnelle sont appréhendés au moyen d'un questionnaire. Il s'avère que les enseignants ont peu investi ce domaine. Les résultats sont discutés par une mise en perspective anthropologique entre technique et écriture et en s'interrogeant également sur l'enseignement de l'écriture de haut niveau en milieu universitaire.
\end{abstract}

\section{ABSTRACT}

Writing digitalized texts and hypertexts require some discursive skills that come with training. First, the introduction of writing technology and its consequences on teaching writing is briefly recalled. Second, the emphasis of the article is put on writing skills developed while one has to integrate hypertexts in an article or a book. Issues of voice, agency, authorship, relevance and interaction between writers and readers are discussed and considered central to the teaching of advanced writing. But who will teach those new skills? This question is addressed in the third part, in which a survey among literacy teachers in Marseille reveals that very few have invested this area. The results indicate a gap between literacy teacher's interests and skills as to writing technology and the need to develop these skills among their students.

* EHESS et CNRS, Centre Norbert Elias.

** Université de Sherbrooke. 
Depuis la naissance de l'ingénierie des formes textuelles (gestion informatisée des documents écrits comprenant notamment les normes iso des caractères et les logiciels de traitement de texte), les sociétés contemporaines se caractérisent par une surabondance de l'information, comme le soulignait le directeur de la BNF, B. Racine (Le Monde, 21 juillet 2008). L'écrit est omniprésent et si l'invention de l'imprimerie est à la source de la démocratisation de l'accès à la lecture, les textes virtuels (textes numérisés et hypertextes) devraient dans les années à venir démocratiser l'accès à l'écriture. Il faudrait néanmoins que les technologies de l'information soient enseignées et que l'écriture fasse l'objet d'un apprentissage dépassant les frontières de la grammaire normative. En effet, il s'agit d'appréhender l'organisation du document écrit dans sa complexité. La démocratisation de l'accès à l'écriture suppose la valorisation à l'école, de ce que Bautier (2010) appelle la littératie étendue :

La littératie étendue désigne les possibilités intellectuelles développées par l'usage heuristique de l'écrit, de l'écrit d'élaboration, celui qui suppose aussi l'interprétation du lecteur, qui n'est ni dans une transparence par rapport au réel, ni une consignation de l'oral. La littératie étendue correspond à ces usages de l'écrit qui, au cours du temps, ont contribué aux constructions et activités intellectuelles qui élaborent des lois générales et non des seules narrations d'expériences locales et spécifiques, qui transforment les expériences en savoirs, qui permettent de tisser ensemble (de penser et d'écrire avec) différentes voix d'origines diverses, savantes et vulgaires, orales et écrites.

Les enjeux sociaux de la littératie étendue sont déjà là : savoir lire et écrire pour produire des connaissances constitue une compétence minimale et attendue pour obtenir un emploi de qualité. De surcroit, lire et écrire prennent place aujourd'hui dans des environnements multimédia, ce qui hausse d'un cran la complexité des processus cognitifs mise en œuvre et qui augmente la charge de savoirs instrumentaux devant être maitrisés. Les rapports au savoir écrire et les rapports à la profession se trouvent par conséquent modifiés par ces nouvelles technologies. Si l'on fait une analogie avec le calcul, les outils de calcul ont également été sources d'innovation et d'amélioration des abaques, en passant successivement des tables à calcul aux bouliers, machines à calculer et enfin, à la première calculatrice en 1972. Les débats révélant la complexité du problème ont accompagné l'emploi de la calculette en classe et finalement, les programmes de 1995 ont stipulé qu'il était nécessaire, en cycle 3 de l'école élémentaire, de savoir utiliser une calculatrice. Dans 
le domaine de l'écrit, par-delà l'apprentissage du code alphabétique, nous faisons l'hypothèse que ces nouvelles technologies induisent des rapports nouveaux non seulement à la gestion des connaissances mais aussi à la gestion de l'apprentissage de l'écriture de haut niveau. Y a-t-il trace de ces préoccupations chez les futurs professeurs de lettres en quatrième année d'université ? Des formations sont-elles envisagées pour appréhender cette nouvelle réalité technique?

Dans cet esprit, nous nous sommes interrogées sur l'appropriation des textes numérisés par des stagiaires professeurs en lettres, en France, en situation d'enseignement. Nous rappellerons, dans une première partie, les enjeux théoriques posés par ce nouveau matériau et nous repérerons les volontés politiques en matière d'éducation à ces nouveaux supports. Dans une deuxième partie, nous présenterons les attitudes des stagiaires et les questions soulevées par ces résultats.

\section{Les textes numérisés et les hypertextes}

\section{Rappel historique}

Les grands principes de l'hypertexte ont été proposés en 1945 par un scientifique américain, Vannevar Bush, conseiller du président Roosevelt. C'est en analysant les difficultés de la transmission des connaissances et les potentialités de l'ordinateur que Vannevar Bush a conseillé de substituer aux méthodes linéaires, un modèle associatif fonctionnant à l'image de l'esprit humain. En 1965, Ted Nelson offre le mot «hypertexte» et est l'initiateur du projet Xanadu, système planétaire d'hypertexte. Des développements expérimentaux voient le jour à partir des années 1980 dans les universités américaines et des programmes sur micro-ordinateur sont disponibles à partir de 1986.

Cette révolution de support d'écriture et de gestion des informations conduit Herrenschmidt (2007) à envisager cette nouvelle technologie comme une révolution américaine. Elle propose l'expression «écriture réticulaire» pour rendre compte de cela. Il s'agit d'une nouvelle étape dans la gestion de l'écriture car, écrire en gérant un texte et des hypertextes, c'est écrire en réseau, c'est penser en arborescence. Toute écriture est définie comme activité de gestion de contraintes (Alamargot et Chanquoy, 2001 ; Olive et Piolat, 2003; Plane, 2006) : contraintes linguistiques, psycholinguistiques, procédurales, rhétoriques, pragmatiques et situationnelles. La planification et la révision d'un document sur support multimédia et comprenant des hypertextes exigent du scripteur 
des macrohabiletés textuelles et discursives qui ne surgiront pas spontanément en apprenant le fonctionnement de logiciels de «design» de documents. La compréhension du processus d'écriture dans tous ses aspects est un préalable, d'autant que la difficulté de lire un document multimédia avec des liens hypertextes est plus grande : plus complexe est l'arborescence de la structure du document, plus exigeante en est sa lecture. Il s'ensuit que l'enseignement du processus d'écriture doit être envisagé en contexte multimédia et d'hypertextes pour qu'il y ait véritablement apprentissage.

\section{Les Technologies de l'information et de la communication (TIC)}

Le maniement des TIC et donc des hypertextes fait partie du socle commun du référentiel des compétences professionnelles pour la formation des personnels d'encadrement au niveau national, en France. Il existe des certifications en TIC par niveau scolaire, puis des formations à l'université et des formations pour les enseignants intervenant auprès des jeunes.

À titre d'exemple, le B2i du primaire est validé, par une équipe enseignante, si les bases de l'outil informatique (à savoir, la bureautique comme le maniement de la «souris», du clavier, etc.) sont maitrisées, si le jeune a été sensibilisé à une attitude citoyenne face aux informations véhiculées par Internet. Doivent être également validés, des items portant sur la réalisation d'un document à l'aide d'un logiciel de traitement de texte, et portant aussi sur la capacité de se documenter par la gestion des produits multimédia.

Au collège, dans le cadre du B2i, la gestion du traitement de texte est prioritaire tant dans les capacités d'élaboration d'un texte que dans sa présentation et sa diffusion au sein d'un texte numérisé ou d'un hypertexte. L'élève doit être capable de réaliser le maniement des ressources documentaires sur supports informatiques, audiovisuels et multimédia. L'attitude critique et responsable constitue aussi des items d'évaluation. L'élève doit faire preuve de discernement entre les sources d'informations accessibles sur Internet. Il doit également comprendre les enjeux du «copier-coller» à partir de documents saisis sur Internet. Là aussi, l'équipe enseignante doit valider le B2i de chaque élève.

Enfin, le rapport Fourgous du 15 février 2010, remis au ministre de l'Éducation nationale, préconise la connexion de $100 \%$ des établissements d'ici 2012, la généralisation des tableaux numériques et la formation des enseignants aux outils. Les TIC sont donc portées par une volonté politique affichée. 
Cependant le texte numérisé n'est pas simplement une nouvelle «cosmétique» technique. Pour reprendre Jeanneret (2000), trois dimensions sont à prendre en compte. Tout d'abord, effectivement, les pouvoirs politiques assurent la diffusion des données, par la mise en place matérielle et par la formation qui s'y rapporte, sur un territoire donné, la France. Ensuite, des acteurs appliquent cette technologie au service des circuits documentaires. C'est le cas du développement des pages web de nombreux organismes. Enfin, les réseaux culturels et traditionnels qui légitimaient les savoirs sont modifiés (par exemple, avec une encyclopédie gratuite, en ligne et enrichie par les lecteurs eux-mêmes). Pour les deux derniers points, l'écrit est amplifié, nécessitant des compétences nouvelles tant pour le lecteur que pour le rédacteur. Dans sa revue de littérature sur les effets des nouvelles technologies sur l'apprentissage de la rédaction, MacArthur (2006, p. 257) fait référence à une évaluation de l'utilisation de l'hypermédia dans le but de favoriser l'émergence de commentaires critiques sur des textes littéraires. Des élèves du secondaire ont appris à manipuler textes et hypertextes et à ajouter des images, ce faisant, résume-t-il, «they created juxtapositions that involved reinterpreting the texts, questioning the underlying assumptions, and reflecting on culture and ideology». De nouvelles compétences émergent donc en écriture.

Les nouvelles technologies d'écriture ont un impact nécessitant des formations qui rendraient explicites ces nouvelles compétences. Pour appréhender le contenu de ces formations, il nous faut maintenant repérer les enjeux linguistiques induits par cette langue écrite sur un nouveau support.

\section{Les enjeux pragmatiques et énonciatifs}

Les textes numérisés et les hypertextes sont des textes virtuels et constituent, nous l'avons souligné en introduction, un enjeu technique et culturel. L'hypertexte se caractérise par un texte numérisé initial auquel sont associés des documents comme des notices, des commentaires, des liens vers d'autres ouvrages, mais aussi des documents sonores, des images et des documents audio-visuels. Les textes numérisés et les hypertextes relèvent d'une technologie nécessitant la connaissance d'un logiciel de traitement de texte. Cependant, il serait réducteur de considérer cette nouvelle technologie de l'écrit comme extérieure à la pratique de la langue écrite. S'il s'agit bien d'un nouvel enjeu technique, alors il est de fait culturel, car «le monde auquel nous nous 
affrontons dans l'expérience la plus quotidienne, est culturel, c'està-dire technique et technicisé de part en part. Nous n'avons aucune expérience naturelle du monde et de nous-mêmes» (Beaune, 1972, p. 5). Plus récemment, Mackey (1988) indique qu'il est aujourd'hui largement admis que la signification d'un élément dépend du contexte dans lequel il figure et que le cadre culturel est le fondement de toute compréhension. Les rapports entre la forme linguistique et la fonction langagière dans un contexte donné et à l'intérieur d'une culture définie permettent d'appréhender dans un document écrit, les quand, où et quoi de la situation. Ils déterminent le choix d'un élément de langue plutôt qu'un autre. Il y a un effet cumulatif et interactif car ces entrelacs sont assujettis, à leur tour, aux contraintes de la culture dont la langue est à la fois une composante et une interface. Jeanneret (2000), également, attire l'attention sur le fait de cette indissociabilité entre pratiques culturelles et techniques des hypertextes, tout comme le recto et le verso d'une feuille de papier.

De plus, du point de vue pragmatique, Rabatel (2008) montre l'effacement de la source énonciative au singulier. Les hypertextes créent un espace énonciatif où les acteurs sont scripteurs et lecteurs par rapport aux autres et par rapport à eux-mêmes.

On a donc affaire à une médiatisation supplémentaire par rapport à l'écrit, inscrite dans de nouvelles pratiques culturelles : l'écran, la gestion de la mise en page, le déplacement des paragraphes par la technique du «copier-coller», l'écriture avec les deux mains sur le clavier, etc.

Ces hypertextes ne peuvent pas exister en l'état sous forme imprimée, car ils constituent en fait une édition critique en constante évolution (Souchier, 1997). En effet, la possibilité d'ajouter des nœuds et des liens spécifiques concrétise l'activité interprétative du lecteur/écrivain. Ces documents virtuels permettent de réintroduire la glose à l'intérieur d'un document écrit et ainsi, la pratique énonciative éditoriale (Souchier, 1997). Nous avons alors des moyens hétérogènes pour élaborer la pensée. Dans un cadre pragmatique, pour reprendre Rabatel (2000), les lecteurs-scripteurs sont dans une dimension plurielle et multi-tâches : il y a en effet, par les hypertextes, des sous énonciateurs et des sur énonciateurs et dans le maniement technique, le scripteur est en même temps, scripteur et lecteur, générant par ce fait de la polyphonie et l'émergence de la dimension auto-dialogique.

Ceci est particulièrement pertinent pour les hypertextes littéraires. En effet, la glose est une ancienne tradition rhétorique où le passage de la transmission orale à la transmission écrite a nécessité une interaction 
dialogique propre au discours. La glose a consisté à rendre intelligible un texte à l'aide de notes et de commentaires portés sur le document luimême. Plusieurs types de glose étaient possibles : celle de l'auteur qui révisait son document, y ajoutant ainsi des éléments additionnels, celle du correcteur qui ajoutait des précisions, commentait afin d'améliorer la diffusion et/ou la compréhension du document, et enfin celle du lecteur qui consignait des notes rendant compte de son activité interprétative.

Que la glose soit celle du rédacteur, du relecteur-éditeur ou du lecteur, il y avait une pratique interactive et énonciative entre l'information écrite et les lecteurs-écrivains. Progressivement, le document écrit s'est substitué intégralement à la glose, jusqu'à condamner l'écriture sur le livre («on ne doit pas salir un livre»!). Avec les hypertextes, nous sommes de nouveau dans une interaction dialogique et polyphonique. Comme l'indique Anis (1998, p. 269), jamais auparavant on n'avait dialogué en direct par écrit avec des inconnus. Il y a ainsi une pratique discursive avec hypertexte avec d'autres lecteurs autour d'un même auteur. Nous pensons que si l'imprimerie a été à l'origine de la démocratisation de la lecture, nous assistons avec l'écriture réticulaire à la démocratisation de l'écriture. Une des conséquences déjà visible est la surabondance de documents écrits, posant des défis d'archives considérables.

Ce changement culturel est-il appréhendé par les enseignants de lettres? Nous pensons que l'avis des professionnels de l'écrit devrait nous permettre d'avoir des éléments de réponses.

Nous avons suivi des enseignants de lettres en stage en vue d'obtenir une certification C2i2e. Cette formation est une option proposée aux stagiaires. Nous souhaitions par des questionnaires rendre compte de leur pratique et/ou de leur réflexion sur l'enseignement de l'écrit avec les technologies de l'écrit.

\section{Rapport aux textes numérisés de vingt professeurs de lettres en formation}

Vingt professeurs stagiaires, «néo-recrutés » comme enseignants, ont participé à cette étude. Cette dernière se déroulait en début et en fin de formation de $\mathrm{C} 2 \mathrm{i} 2 \mathrm{e}$. Ces professeurs stagiaires en collège étaient certifiés en lettres modernes, avaient entre 25 et 32 ans et suivaient une formation TICE afin d'acquérir la certification C2i2e qui, en 2009, était une option dans le cadre de leur formation en alternance. Cette certification permettait, et permet toujours, de valider les compétences des 
élèves dans le secondaire (B2i). Ces stagiaires avaient tous au moins une licence en lettres modernes et enseignaient dans l'académie d'AixMarseille. Durant cette formation, ils avaient à la fois une formation générale sur les enjeux juridiques de ces nouveaux outils et une formation spécifique sur la construction d'hypertextes et de diaporamas. Le propos de notre recherche était d'une part de repérer comment ils se positionnaient dans leur pratique personnelle par rapport à ces nouvelles technologies et d'autre part de rendre compte de l'impact de ces dernières sur leur activité professionnelle.

En début de stage, ils ont répondu individuellement et par écrit, à un questionnaire comprenant 6 questions (tableau 1). Après avoir rendu le questionnaire, il y a eu un échange de points de vue entre les stagiaires pendant une heure, en présence de l'enquêtrice. Au terme de la formation, ils devaient présenter un travail réalisé avec un support numérique (diaporama et/ou texte numérique avec maniement des outils de gestion de traitement de texte et/ou maniement du logiciel didapage).

\begin{tabular}{|l|l|l|l|l|}
\hline Questionnaire en début de stage & Jamais & Parfois & Souvent & Toujours \\
\hline $\begin{array}{l}\text { 1. Utilisez-vous un traitement de texte } \\
\text { pour préparer un cours? }\end{array}$ & & & & \\
\hline 2. Utilisez-vous un diaporama? & & & & \\
\hline $\begin{array}{l}\text { 3. Transmettez-vous vos plans de cours } \\
\text { par Internet à vos élèves? }\end{array}$ & & & & \\
\hline $\begin{array}{l}\text { 4. Donnez-vous des sitographies à vos } \\
\text { èlèves? }\end{array}$ & & & & \\
\hline $\begin{array}{l}\text { 5. Donnez-vous des exercices de } \\
\text { traitement de texte? }\end{array}$ & & & & \\
\hline 6. Réservez-vous la salle informatique? & & & & \\
\hline
\end{tabular}

Tableau 1. - Questionnaire transmis aux stagiaires.

Les données sont traitées en suivant l'ordre chronologique des questions.

\section{Utilisez-vous un traitement de texte pour préparer un cours?}

Onze enseignants n'en emploient jamais, quatre enseignants en utilisent un occasionnellement et cinq enseignants s'en servent souvent. Cela montre que ces vingt stagiaires ne s'approprient pas l'écriture numérisée. Lors de la discussion, la majorité d'entre eux pense que les traitements de texte relèvent strictement du domaine technique. Ils confirment leur propos en ne validant pas les items du B2i des élèves au 
sein du collège où ils travaillent, considérant que cette certification relève du domaine technique.

\section{Utilisez-vous un diaporama?}

Seize enseignants n'en emploient jamais, deux enseignants le font occasionnellement et deux enseignants s'en servent toujours. Même si le diaporama est un support ne remettant pas fondamentalement en cause la gestion de l'écrit, la position des enseignants est du même ordre et révèle une attitude réservée devant une nouvelle technologie.

\section{Transmettez-vous vos plans de cours par Internet à vos élèves?}

Aucun enseignant stagiaire n'a recours à cette pratique. La question a surpris les stagiaires à deux niveaux : lors de la préparation de leur cours, le plan n'est pas numérisé et lors de la transmission de leurs cours, il n'est pas communiqué. Le plan est donc dicté aux élèves.

Pour certains stagiaires, les jeunes n'ayant pas tous accès à Internet, il n'est pas envisageable de créer une inégalité supplémentaire.

\section{Donnez-vous des sitographies à vos élèves?}

Deux enseignants ne le font jamais, dix enseignants le font occasionnellement et huit enseignants souvent. La démarche documentaire numérisée est donc appréhendée par les enseignants-stagiaires et elle est motivée par le fait qu'il faut aider les élèves à se repérer dans les différentes pages accessibles sur Internet.

\section{Donnez-vous des exercices de traitement de texte?}

Aucun enseignant stagiaire n'a cette pratique. Certains refusent des documents rendus dactylographiés. Une des explications pourrait être liée à la préparation aux épreuves du baccalauréat de français. Le regard sur les hypertextes et les textes numérisés est uniquement technique : par défaut, il n'y aurait pas d'incidence entre la technologie employée et l'acte d'écrire et de lire.

\section{Réservez-vous la salle informatique?}

Quinze enseignants ne le font jamais, deux enseignants le font occasionnellement et deux enseignants souvent; un enseignant affirme la réserver toujours. Les réponses reflètent les pratiques des vingt enseignants-stagiaires. En effet, pourquoi réserver une salle avec des ordinateurs si les textes numérisés et les hypertextes ne sont pas appréhendés?

À la fin du stage, afin de valider leur formation en C2i2e, les stagiaires ont tous réalisé un diaporama sur un thème littéraire, mais ils n'ont pas investi le traitement de texte. Ils ont, pour trois d'entre eux, pris connaissance dans le maniement d'un logiciel qui permet de fabriquer un livre hypertexte (logiciel didapage). 


\section{Discussion et perspective}

Il y a donc une nouvelle écriture, mais elle se situe pour l'instant dans la constitution de la glose. L'attitude des enseignants-stagiaires, même sur un petit échantillon, interpelle. Le comportement culturel des acteurs face à une nouvelle technologie montre que cette dernière n'est pas intégrée comme modifiant notre rapport à l'écriture. En effet, par-delà cette étude, les observations empiriques réalisées par différents formateurs pour évaluer les pratiques de classe des professeurs stagiaires ont tendance à confirmer ces postures.

Il y a quinze ans, Dubois (1995) avait déjà attiré l'attention sur l'intérêt de l'emploi de "l'informatique» dans le cadre de l'étude d'une œuvre et dans la lecture-écriture du texte argumentatif. Les résultats que nous obtenons interrogent et soulèvent un problème culturel d'appropriation technologique dans un champ, la littérature, qui est peu problématisé (à la différence de la physique ou des mathématiques). En cela, la francophonie accuse un retard en regard du monde anglosaxon, où l'enseignement des TIC intégré à l'enseignement de la rédaction est documenté et observé depuis au moins 15 ans (Beaufort, 2006; MacArthur, 2009).

Abdallah (2001, p. 151) rappelle que si les techniques sont des ensembles de savoirs matérialisés, il est nécessaire de les considérer en même temps comme «des ensembles de normes organisées». D'après cet auteur, «le recentrement des normes par les utilisateurs des techniques dans le processus d'apprentissage est la modalité particulière par laquelle se réalise l'appropriation des techniques». Or, il y a bien des normes à légiférer dans ce nouveau maniement de l'écrit. Par exemple, la gestion de l'opération «copier-coller» empêche désormais le lecteur de demeurer analytique et certaines erreurs de logique et/ou de structures de phrases peuvent ne pas être appréhendées. La pratique des liens au sein des hypertextes présuppose également des compétences argumentatives qui reposent sur des normes de cohérence et de cohésion. Enfin, le sens n'est pas dans la technique mais dans la pratique sociale partagée à l'aide de cette technique. Or, l'investissement dans l'écriture réticulaire peut remettre en cause, dans un premier temps, une «souplesse dans le maniement du savoir» (Laforest, dans ce volume). Cette souplesse qui caractériserait le professionnel performant serait mise à mal au moment de l'appropriation par les enseignants de l'écriture réticulaire. Dans le domaine de l'écrit, la séparation traditionnelle entre les imprimeurs et les lettrés a conduit les enseignants de lettres à moins 
se positionner sur les rapports entre écriture et supports techniques de l'écriture et, par conséquent, à moins acquérir cette souplesse, ne seraitce que le maniement expert des touches du clavier.

Cela fait également écho aux propos de Blanc (dans ce volume) sur le savoir de conviction: on est bien là dans une certaine représentation de la technologie considérant le contenu (le sens véhiculé par l'écrit) comme dissocié du contenant (hypertextes et textes numérisés). Bien sûr, toute forme d'écriture suppose une part de connaissances procédurales. Cependant, il n'en demeure pas moins que rédiger dans un environnement multimédia ne met pas en sourdine la difficulté d'écrire. Écrire s'appuie sur des processus cognitifs de haut niveau : le scripteur doit gérer la cohérence de son propos et en favoriser la figurabilité. Pour parvenir à cette fin, il doit selon de nombreux auteurs (Hayes et Flower, 1980; Bereiter et Scardamalia, 1987; Hayes, 1996; Alamargot et Chanquoy, 2001) :

- maitriser son sujet en profondeur (connaissances du thème);

- posséder des connaissances avancées sur le langage et les règles et conventions d'écriture (lexique, grammaire, syntaxe);

- comprendre le processus d'écriture comme un processus de résolution de problème en y associant des modèles de production de textes.

Les hypertextes et les textes numérisés créent un environnement de tâches rédactionnelles plus complexe et obligent le scripteur à maintenir un niveau de métacognition très élevé. Il est nécessaire en effet de développer des capacités de synthèse pour créer des hyperliens pertinents et, en même temps, une expansion textuelle avec des éléments hiérarchisés. Pour ce dernier point, il ne s'agit pas d'une propension aux opinions de toutes sortes, mais d'une construction polyphonique alliant argumentation, information, description et narration. L'enjeu alors est de s'appuyer sur la structuration de la langue écrite telle que nous la connaissons par notre expérience des décennies antérieures et de la revisiter, en intégrant sa dimension réticulaire.

Le paradoxe est maintenant le suivant : les stagiaires en lettres doivent enseigner aux élèves à lire et à écrire des textes, notamment littéraires, dans un environnement multimédia, mais ils dissocient l'environnement technologique où s'inscrit la textualité des contenus dispensés. Ceci revient à défendre qu'ils ne considèrent pas l'écriture réticulaire comme un processus qui s'enseigne. Mais l'institution formatrice est aussi à interroger : quels sont les supports d'enseignement en licence 
de lettres modernes? Comment est envisagé l'accès à l'écriture réticulaire? Est-elle appréhendée comme une simple certification, parallèle aux études? Les mémoires de recherche intègrent-ils cette dimension dans les contraintes rédactionnelles? Comme on le voit, l'enquête serait à poursuivre dans le milieu universitaire, notamment en suivant l'impact de la mastérisation de la formation des enseignants. Si ce changement de support n'est pas conceptualisé par les enseignants universitaires de lettres, le risque à moyen terme serait que les enseignants de français deviennent les illettrés de demain et que les enseignements soient essentiellement des cours sur la structure linguistique (enseignement du code linguistique et moins du processus d'écriture dans toute sa complexité, étant donné le passage rapide de l'imprimé au multimédia auquel nous assistons). Dans le contexte contemporain où la formation des enseignants traverse une crise majeure, toute discipline confondue, il est à craindre que la centration de l'enseignement sur les microhabiletés textuelles n'apparaisse comme dérisoire.

Cependant, au-delà de la formation proprement dite, des initiatives émergent en France, comme la formation de rédacteur professionnel, indiquant par ce fait de nouvelles formes de professionnalité et aussi une science de l'écrit en devenir. Car, comme le dit Herrenschmidt (2007, p. 453),

un CD Rom peut enregistrer 600 millions d'octets soit 1000 livres de 400 pages à raison de 1500 caractères typographiques par page. Un DVD de 12 centimètres carrés peut actuellement contenir 15 milliards d'octets, soit 25000 livres. C'est admirable mais au-delà des capacités de lecture d'un être humain. Les ordres de grandeur de ces supports mémoriels informatiques n'entretiennent de rapport ni avec ce qui a précédé ni avec les capacités des vivants.

Nos idées de support de l'écrit sont donc complètement dépassées et nécessitent donc de nouveaux concepts et de nouveaux comportements pour les années à venir dans la formation des professeurs de lettres.

\section{RÉFÉRENCES BIBLIOGRAPHIQUES}

Abdallah N. (2001) : Techniques et cultures, Comment s'approprie-t-on des technologies transférées?, Toulouse, Octares éd.

Alamargot D. et Chanquoy L. (2001): Through the models of writing, Dordrecht, Kluwer Academic Publishers. 
ANIs J. (1998): Texte et ordinateur. L'écriture réinventée?, Paris, Bruxelles : De Boeck \& Larcier.

ARSAC J. (1987) : Les machines à penser. Des ordinateurs et des hommes, Paris, Seuil.

BAUTIER E. (2008) : «Apprendre à écrire et/ou entrer dans la littératie étendue?», dans Actes de colloque international De la France au Québec : l'écriture dans tous ses états (Université de Poitiers, 12-15 novembre 2008).

- (2009) : «Quand le discours pédagogique entrave la construction des usages littéraciés du langage», Pratiques : écrits et savoirs, $\mathrm{n}^{\circ}$ 143-144, p. 11-26.

Beaufort A. (2006) : «Writing in the Professions», dans Peter Smagorinsky (éd.), Research on Composition. Multiple Perspectives on two Decades of Change, p. 217-243.

BeAune J.-C. (1972) : La technologie, Paris, PUF.

Bereiter C. et Scardamalia M. (1987) : The Psychology of Written Composition, Hillsdale (NJ), Lawrence Erlbaum Associates.

Dubors Cl. (1995) : «Informatique et enseignement littéraire», EPI, n 80 , p. 73-79.

HAYes J.R. et Flower L.S. (1980) : «Identifying the organization of writing processes», dans L. W. Gregg et E. R. Steinberg (éds), Cognitive Processes in Writing, Hillsdale (NJ), Lawrence Erlbaum Associates, p. 3-30.

HAYES J. R. (1996) : «A new framework for understanding cognition and affect in writing», dans M. Levy et S. Randell (éds), The Science of Writing. Theories, Methos, Individual Differences, and Applications, Mahwah (NJ), Lawrence Erbaum Associates, p. 1-28.

HerRenschmidt Cl. (2007) : Les trois écritures Langue Nombre Code, Paris, Gallimard.

JAFFRÉ J-P. (2003) : «L'écriture et les nouvelles technologies : ce que les unes nous apprennent de l'autre», Quatrièmes rencontres Réseaux humains / réseaux technologiques, Université de Poitiers.

JEANNeRET Y. (2000) : Y a-t-il (vraiment) des technologies de l'information?, Lille, Presses universitaires du Septentrion.

MacArthur C. (2009) : «Technology and struggling writers: A review of research», dans V. Connelly, A. L. Barnett, J. Dockrell et A. Tolmie (éds), Teaching And Learning Writing, British Journal of Educational Psychology, Monograph Series II, n ${ }^{\circ}$ 6, p. 159-175.

Mackey W. F. (1988) : «Texte, Contexte et Culture, Traduction, Terminologie», Rédaction, vol. 1, p. 11-20.

Olive T. et Piolat A. (2003) : «Activation des processus rédactionnels et qualité des textes», Le langage et l'Homme, vol. 38, n 2, p. 191-203. 
Plane S. (2006) : «Singularités et constantes de la production d'écrit. L'écriture comme traitement de contraintes», dans J. Laffont-Terranova et D. Colin (éds), Didactique de l'écrit. La construction des savoirs et le sujet écrivant, Namur (Belgique), Presses universitaires de Namur, p. 33-45.

Rabatel A. (2000) : «Un, deux, trois points de vue? Pour une approche unifiante des points de vue narratif et discursif », La lecture littéraire, $\mathrm{n}^{\circ} 4$, p. $195-254$.

- (2008) : Homo Narrans. Pour une analyse énonciative et interactionnelle du récit, Limoges, Lambert-Lucas.

SOUCHIER E. (1997) : Lire \& écrire : éditer. Des manuscrits aux écrans, mémoire pour l'HDR, université Paris 7.

SOUChier E et JEANNeret Y. (2005) : «Médias informatisés et énonciation éditoriale», Les écritures d'écran : histoire, pratiques et espaces sur le Web (colloque des mercredi 18 et jeudi 19 mai 2005, Aix-en-Provence, Maison méditerranéenne des sciences de l'homme).

Souchier E., Jeanneret Y et Le Marec J. (2003) : Lire, écrire, récrire : objets, signes et pratiques des médias informatisés, Paris, BPI. 